\title{
Comunidade de Odonata (Insecta) em áreas naturais de Cerrado e monocultura no nordeste do Estado de São Paulo, Brasil: relação entre o uso do solo e a riqueza faunística
}

\author{
Patrícia S. Ferreira-Peruquetti ${ }^{1} \&$ Alaíde A. Fonseca-Gessner ${ }^{1}$
}

${ }^{1}$ Departamento de Hidrobiologia, Universidade Federal de São Carlos. Caixa Postal 676, 13565-905 São Carlos, São Paulo, Brasil.E-mail: patricia@iris.ufscar.br

\begin{abstract}
Odonata community on natural areas of Cerrado and monoculture of northeastern São Paulo State, Brazil: relationship between land use and richness. Stream ecosystems may be strongly influenced in different ways by land use. The effects of land-cover patterns on Odonata community were studied in eight streams and seven lakes-dams of northeastern São Paulo State. The pattern analyzed was monoculture (sugar cane) vs. nature reserve in terms of Odonata species richness and similarity. Eighty-five Odonata species were sampled. The species richness was higher in the monoculture (33 species) than nature reserve (30 species) and 22 species were common to both areas. There was not verified relationship between land use and physical or chemical characteristics of the streams or lakes-dams water. These results suggest that some Odonata species are benefited and other affected by anthropogenic actions, although more studies are necessary to evaluate this hypothesis. There were ten new records for São Paulo State (Neoneura sylvatica, Coryphaeschna adnexa, Erythemis haematogastra, E. mithroides, Micrathyria longifasciata, M. pseudeximia, Planiplax machadoi, Lauromacromia sp., Tibiagomphus sp. and Aeschnosoma sp.).
\end{abstract}

KEY WORDS. Cerrado, diversity, land use, Odonata, species richness.

A manutenção da biodiversidade dos cursos d'água diante do desenvolvimento humano tem recebido grande atenção nos últimos anos (AlLAN \& FleCKer 1993). Várias pesquisas têm demonstrado que a conversão de florestas em pastagens ou áreas residenciais pode influenciar os habitats e as comunidades aquáticas de várias maneiras (GurTz \& Wallace 1984, Lenat \& Crawford 1994, Омeto et al. 2000, Sponseller et al. 2001). Por isso, conhecer a relação da biota aquática com o uso do solo do entorno é de grande importância e oferece suporte na elaboração de estratégias regionais de manejo (AlLAN \& JOHNSON 1997).

As libélulas são insetos com fases larval aquática e adulto aéreo. Possuem ciclo de vida longo, com a fase larval podendo alcançar até dois anos (CORBET 1980), portanto estando sujeitas às alterações ambientais. São consideradas potenciais indicadoras da qualidade do habitat e, apesar de serem menos sensíveis que outros insetos aquáticos, são conspícuas, facilitando os diagnósticos rápidos da qualidade da água (CARLE 1979, Schmidt 1985, Gorham \& Vodopich 1992, Samways \& Steytler 1996, Ferreira-Peruquetti \& De Marco Jr. 2002).

Este trabalho mostra a relação do uso do solo (monocultura versus unidade de conservação) com a comunidade de Odonata, sob os aspectos de riqueza e similaridade, em áreas de cerrado. Também são apresentadas novas ocorrências de Odonata para o Estado de São Paulo.

\section{MATERIAL E MÉTODOS}

\section{Área de estudo}

O município de Luís Antônio possui 59.613,63ha e está localizado na região nordeste do Estado de São Paulo $\left(21^{\circ} 20^{\prime}\right.$, $21^{\circ} 55^{\prime} \mathrm{S}$ e $\left.47^{\circ} 35^{\prime}, 47^{\circ} 55^{\prime} \mathrm{W}\right)$. A maior parte do município é ocupada por agroecossistemas $(67,37 \%)$, seguida pelas áreas naturais $(31,7 \%)$ e pelos corpos d'água $(0,93 \%)$ As áreas naturais incluem vegetação natural e semi-natural em vários estados de conservação (PIREs et al. 2000), principalmente na Estação Ecológica Jataí (EEJ).

Localizada entre os paralelos $21^{\circ} 33^{\prime}$ e $21^{\circ} 37^{\prime} \mathrm{S}$ e $47^{\circ} 45^{\prime}$, $47^{\circ} 51^{\prime} \mathrm{W}$, a EEJ têm $4.532,18$ ha e foi criada pelo decreto-lei $\mathrm{n}^{\circ}$ 18.997 (15/06/82, SP) (São Paulo 1985). Na EEJ, encontram-se 15 das dezenas de lagoas marginais do rio Mogi-Guaçu e uma das maiores áreas de cerrado (lato sensu) do Estado de São Paulo (SÃo Paulo 1997).

A área de estudo é caracterizada por duas estações climáticas bem definidas: uma chuvosa, com pluviosidade e tempe-

Revista Brasileira de Zoologia 20 (2): 219-224, junho 2003 
raturas elevadas (de novembro a abril) e outra seca, com pluviosidade e temperaturas mais baixas (de maio a outubro) (Cavalheiro et al. 1990). De acordo com classificação de Koppen, o clima da região é Aw (Setzer 1966).

\section{Amostragem}

As coletas foram conduzidas durante o período de julho de 2000 a março de 2002, abrangendo as estações seca e chuvosa e levando-se em conta os principais usos do solo do município: monocultura (cana-de-açúcar) e a unidade de conservação (EEJ). Foram escolhidos dezoitos pontos de coleta entre os sistemas lótico e lêntico (Fig. 1). Todos os córregos amostrados na EEJ possuiam uma faixa de mata ciliar com mais de $20 \mathrm{~m}$. Na área de monocultura, dois cursos d'água possuiam mata ciliar, com uma faixa de até $5 \mathrm{~m}$.

Para a amostragem das espécies, seguiu-se o método descrito em Ferreira-Peruquetti \& De Marco Jr. (2002). Houve somente modificação da rede utilizada para coleta das larvas (neste trabalho, rede manual tipo "D" com malha de $500 \mu \mathrm{m}$ ).

As amostras foram agrupadas em quatro categorias: (I) unidade de conservação - sistema lótico (354 amostras); (II) unidade de conservação - sistema lêntico (326 amostras); (III) monocultura - sistema lótico (324 amostras) e (IV) monocultura - sistema lêntico (215 amostras).

\section{Análises}

Para verificar a possível influência do uso do solo sobre a qualidade d'água, foram registrados os valores de temperatura, oxigênio dissolvido, $\mathrm{pH}$ e condutividade elétrica, utilizandose um sensor múltiplo. Como estas variáveis físicas e químicas são muitas vezes interdependentes (Esteves 1998), elas foram agrupadas utilizando-se a Análise de Componentes Principais e posteriormente testada a diferença entre as quatro categorias acima citadas, tomadas duas a duas, pelo teste $t$ (MANLY 1994).

Para verificar a possível influência do uso do solo sobre a comunidade de Odonata, foi estimada a riqueza em espécies pelo método Jackknife (Heltshe \& Forrester 1983, Colwell \& CodDington 1994) em cada categoria estabelecida. O intervalo de confiança foi estimado para a riqueza em espécies, pelos métodos normalmente utilizados (MANLY 1991).

A diversidade $\beta$ foi calculada pela versão modificada do índice de similaridade Morisita-Horn (Wolda 1983). Este é considerado uma das mais apropriadas medidas de diversidade, sendo relativamente pouco influenciado pela riqueza e tamanho da amostra, apesar de ser sensível a abundância das espécies com maior número de indivíduos (MagurRan 1988).

Para o cálculo do índice de similaridade e da estimativa da riqueza foi utilizado o programa Estimates (Versão 6, ColwELl 1997).

\section{RESULTADOS}

No município de Luís Antônio foram registradas 85 espécies de Odonata, 22 ocorreram tanto na EEJ como na área de monocultura, 33 somente na monocultura e 30 somente na EEJ. Há dez novas ocorrências para o Estado de São Paulo: Neoneura sylvatica, Coryphaeschna adnexa, Erythemis haematogastra, E. mithroides, Micrathyria longifasciata, $M$. pseudeximia, Planiplax machadoi, Lauromacromia sp., Tibiagomphus sp., Aeschnosoma sp. (Tab. I).

Argia lilacina, as espécies de Oxyagrion, Mnesarete pudica

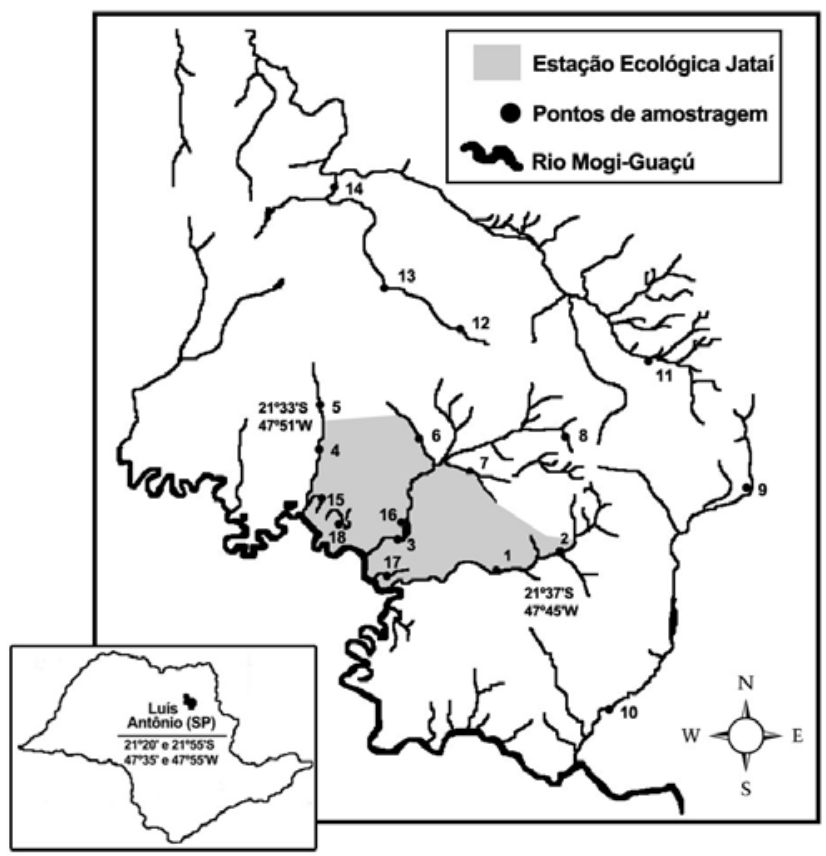

Figura 1. Localização da Estação Ecológica Jataí (área preenchida) no município de Luís Antônio, São Paulo, Brasil. Os números indicam os pontos de coleta: (1-2) Córrego Cafundó; (3) Córrego Beija-Flor; (4-5) Córrego Boa Sorte; (6) Córrego Jordão; (7) Córrego Cabaça; (8) Represa da Serra; (9) Ribeirão Vassununga, trecho represado; (10) Ribeirão Vassununga; (11) Ribeirão da Onça; (12) Córrego Volta Grande, trecho represado; (13-14) Córrego Volta Grande; (15) Lagoa do Óleo; (16) Represa Beija-Flor; (17) Lagoa do Diogo; (18) Lagoa do Quilômetro. Modificado da carta gerada a partir do banco de dados digitais georeferenciados do Laboratório de Planejamento Ambiental do Departamento de Hidrobiologia da UFSCar.

pudica, Brachymesia herbida, Dasythemis venosa, Diastatops obscura, Erythrodiplax anomala, E. media, E. paraguayensis, Macrothemis musiva, Micrathyria catenata, M. longifasciata, M. ocellata dentiens, M. pirassunungae, Orthemis discolor, Perithemis mooma, Tauriphila argo, Tramea abdominalis, T. calverti, Zenitoptera lanei e Lestes bipupillatus ocorreram somente na área de monocultura (Tab. I).

Heliocharis amazona ocorreu somente nos córregos da EEJ. Outras espécies também só foram encontradas na unidade de conservação: Cyanallagma nigrinuchale, Homeoura lidneri, Ischnura fluviatilis, Telebasis willinki, Lauromacromia sp., Brechmoroga praedatrix, Erythrodiplax ochracea, E. umbrata, Macrothemis imitans imitans, Micrathyria almeidai, M. artemis, M. pseudoeximia, Oligoclada amphinome, O. laetitia, Perithemis lais, Epipleoneura venezuelensis, E. williamsoni, Neoneura bilinearis e N. sylvatica (Tab. I).

Das sete espécies de Erythemis coletadas, cinco ocorreram na EEJ (E. attala, E. credula, E. haematogastra, E. mithroides, E. plebeja), enquanto E. peruviana e E. vesiculosa foram comuns as duas áreas. 
Tabela I. Ocorrência das espécies de Odonata em diferentes usos do solo, estações do ano e sistemas aquáticos, no município de Luís Antônio. (SE) estação seca; (CH) estação chuvosa; (LO) sistema lótico; (LE) sistema lêntico; * fase larval.

\begin{tabular}{cc}
\hline Espécies & Uso do solo \\
\cline { 2 - 2 } & $\begin{array}{c}\text { Unidade de } \\
\text { conservação }\end{array}$ \\
\hline
\end{tabular}

Aeshnidae

Castoraeschna colorata (Martin, 1908) (LE,SE)

Castoreschna sp. (LE,CH)

Coryphaeschna adnexa (Hagen, 1861)* (LO,SE)

Coryphaeschna perrensi (McLachlan, 1887) (LO,LE,CH)

Coenagrionidae

Acanthagrion ascendens Calvert, 1909 (LO,LE, SE, CH)

A. chacoense Calvert, 1909 (LE,SE)

A. gracile (Rambur, 1842) (LO,LE,SE,CH)

A. lancea Selys, $1876(\mathrm{LE}, \mathrm{CH})$

A. minutum (Leonard, 1977) (LE, SE, CH)

A. temporale Selys, 1876 (LE,SE,CH)

A. truncatum Selys, 1876 (LO,LE,SE,CH)

Argia lilacina Selys, 1865 (LO,SE,CH)

A. reclusa Selys, 1865 (LO,SE,CH)

Cyanallagma nigrinuchale (Selys, 1876) (LE,SE)

Homeoura chelifera (Selys, 1876) (LE,SE,CH)

H. lindneri (Ris, 1928) (LE,SE,CH)

Ischnura capreolus (Hagen, 1861) (LE,SE,CH)

I. fluviatilis Selys, 1876 (LE, SE,CH)

Oxyagrion evanescens Calvert, 1909 (LE,CH)

O. microstigma Selys, 1876 (LE,SE,CH)

O. santosi Martins, 1967 (LO,SE)

O. terminale Selys, 1876 (LO,SE,CH)

Telebasis carmesina Calvert, 1909 (LO,LE,SE,CH)

T. limoncocha Bick \& Bick, 1995 (LE,SE,CH)

T. willinki Fraser, 1948 (LE,SE,CH)

Tigriagrion aurantinigrum Calvert, 1909 (LO,LE,SE,CH)

Calopterygidae

Hetaerina rosea Selys, 1853 (LO,SE,CH)

Mnesarete pudica pudica (Hagen in Selys, 1853) $(\mathrm{LO}, \mathrm{CH})$

Corduliidae

Aeschnosoma sp. * $(\mathrm{LO}, \mathrm{CH})$

Lauromacromia sp. * (LO,SE)
Tabela I. Continuação.

\begin{tabular}{|c|c|c|}
\hline \multirow{2}{*}{ Espécies } & \multicolumn{2}{|c|}{ Uso do solo } \\
\hline & $\begin{array}{l}\text { Unidade de } \\
\text { conservação }\end{array}$ & Monocultura \\
\hline \multicolumn{3}{|l|}{ Dicteriadidae } \\
\hline Heliocharis amazona Selys, 1853 (LO,CH) & $\mathrm{X}$ & \\
\hline \multicolumn{3}{|l|}{ Gomphidae } \\
\hline Aphylla spp. (LE,CH) & $\mathrm{X}$ & $\mathrm{X}$ \\
\hline Phyllocycla spp.* (LO,SE,CH) & & $\mathrm{X}$ \\
\hline Progomphus spp.* (LO,SE,CH) & $x$ & $\mathrm{X}$ \\
\hline Tibiagomphus sp. * (LO,SE) & $x$ & $\mathrm{X}$ \\
\hline \multicolumn{3}{|l|}{ Libellulidae } \\
\hline Brachymesia herbida (Gundlach, 1889) (LE,CH) & & $\mathrm{X}$ \\
\hline Brechmorhoga praedatrix Calvert, 1909 (LO,CH) & $x$ & \\
\hline Dasythemis venosa (Burmeister, 1839)* (LO,SE) & & $x$ \\
\hline Diastatops intensa Montgomery, 1940 (LE,CH) & $x$ & $\mathrm{X}$ \\
\hline D. obscura (Fabricius, 1775) (LE, CH) & & $\mathrm{x}$ \\
\hline $\begin{array}{l}\text { Elasmothemis cannacrioides (Calvert, 1906) } \\
(\mathrm{LO}, \mathrm{CH})\end{array}$ & $\mathrm{X}$ & $\mathrm{x}$ \\
\hline Erythemis attala (Selys in Sagra, 1857) (LE,CH) & $\mathrm{X}$ & \\
\hline E. credula (Hagen, 1861) (LE, SE,CH) & $\mathrm{X}$ & \\
\hline E. haematogastra (Burmeister, 1839) (LE, CH) & $x$ & \\
\hline E. mithroides (Brauer, 1900) (LE,SE.CH) & $\mathrm{X}$ & \\
\hline E. peruviana (Rambur, 1842) (LE,SE,CH) & $\mathrm{X}$ & $x$ \\
\hline E. plebeja (Burmeister, 1839) (LE,CH) & $\mathrm{X}$ & \\
\hline E. vesiculosa (Fabricius, 1775) (LE, SE,CH) & $x$ & $x$ \\
\hline Erythrodiplax anomala (Brauer, 1865) (LE,SE,CH) & & $x$ \\
\hline E. fusca (Rambur, 1842) (LO,LE,SE,CH) & $x$ & $\mathrm{X}$ \\
\hline E. latimaculata Ris, 1911 (LE,SE,CH) & $\mathrm{x}$ & $\mathrm{x}$ \\
\hline E. media Borror, $1942(\mathrm{LO}, \mathrm{LE}, \mathrm{SE}, \mathrm{CH})$ & & $\mathrm{x}$ \\
\hline E. ochracea (Burmeister, 1839) (LE, SE,CH) & $\mathrm{x}$ & \\
\hline E. paraguayensis (Förster, 1905) (LE,SE,CH) & & $x$ \\
\hline E. umbrata (Linnaeus, 1758) (LE,CH) & $\mathrm{X}$ & \\
\hline $\begin{array}{l}\text { Gynothemis venipunctata Calvert, } 1909 \\
\text { (LO,SE,CH) }\end{array}$ & $\mathrm{X}$ & $\mathrm{x}$ \\
\hline $\begin{array}{l}\text { Macrothemis imitans imitans Karsch, } 1890 \\
(\mathrm{LO}, \mathrm{CH})\end{array}$ & $\mathrm{X}$ & \\
\hline M. musiva Calvert, $1898(\mathrm{LO}, \mathrm{CH})$ & & $x$ \\
\hline $\begin{array}{l}\text { Miathyria marcella (Selys in Sagra, 1857) } \\
(\mathrm{LE}, \mathrm{CH})\end{array}$ & $\mathrm{X}$ & $\mathrm{X}$ \\
\hline Micrathyria almeidai Santos, 1945 (LE,CH) & $\mathrm{X}$ & \\
\hline M. artemis Ris, 1911 (LE,CH) & $\mathrm{X}$ & \\
\hline M. catenata Calvert, 1909 (LO,LE,CH) & & $x$ \\
\hline M. longifasciata Calvert, 1909 (LO,CH) & & $\mathrm{X}$ \\
\hline
\end{tabular}

Revista Brasileira de Zoologia 20 (2): 219-224, junho 2003 
Tabela I. Continuação.

\begin{tabular}{|c|c|c|}
\hline \multirow{2}{*}{ Espécies } & \multicolumn{2}{|c|}{ Uso do solo } \\
\hline & $\begin{array}{l}\text { Unidade de } \\
\text { conservação }\end{array}$ & Monocultura \\
\hline \multicolumn{3}{|l|}{ Libellulidae } \\
\hline M. ocellata dentiens Calvert, 1909 (LE,CH) & & $\mathrm{x}$ \\
\hline M. pirassunungae Santos, 1953 (LO,LE,CH) & & $\mathrm{x}$ \\
\hline M. pseudeximia Westfall, 1992 (LE,CH) & $\mathrm{x}$ & \\
\hline Oligoclada amphinome Ris, 1919 (LE,CH) & $\mathrm{x}$ & \\
\hline O. laetitia Ris, 1911 (LE,CH) & $\mathrm{x}$ & \\
\hline Orthemis discolor (Burmeister, 1839) (LE,SE,CH) & & $\mathrm{x}$ \\
\hline Perithemis lais (Perty, 1834) (LE,SE,CH) & $x$ & \\
\hline P. mooma Kirby, 1889 (LE,SE,CH) & & $\mathrm{x}$ \\
\hline Planiplax machadoi Santos, 1949 (LE,SE,CH) & $\mathrm{x}$ & $\mathrm{x}$ \\
\hline Rhodopygia sp.* (LE,SE) & & $\mathrm{x}$ \\
\hline Tauriphila argo (Hagen, 1869) (LE,CH) & & $\mathrm{x}$ \\
\hline Tauriphila sp. ${ }^{*}(\mathrm{LE}, \mathrm{SE})$ & $\mathrm{x}$ & \\
\hline Tramea abdominalis (Rambur, 1842) (LE,CH) & & $x$ \\
\hline T. binotata (Rambur, 1842) (LE,SE,CH) & $x$ & $x$ \\
\hline T. calverti Muttkowski, 1910 (LE,CH) & & $\mathrm{x}$ \\
\hline Zenithoptera lanei Santos, 1941 (LE,CH) & & $x$ \\
\hline \multicolumn{3}{|l|}{ Lestidae } \\
\hline Lestes bipupillatus Calvert, 1909 (LE,SE,CH) & & $\mathrm{x}$ \\
\hline L. paulistus Calvert, 1909 (LE,SE) & $x$ & $\mathrm{x}$ \\
\hline \multicolumn{3}{|l|}{ Protoneuridae } \\
\hline $\begin{array}{l}\text { Epipleoneura venezuelensis Rácenis, } 1955 \\
(\mathrm{LE}, \mathrm{CH})\end{array}$ & $\mathrm{x}$ & \\
\hline E. williamsoni Santos, 1957 (LE,SE) & $x$ & \\
\hline Neoneura bilinearis Selys, $1860(\mathrm{LO}, \mathrm{CH})$ & $\mathrm{x}$ & \\
\hline N. sylvatica Hagen in Selys, 1886 (LO,CH) & $\mathrm{x}$ & \\
\hline
\end{tabular}

Apesar da ocorrência de espécies comuns entre as áreas de monocultura e da unidade de conservação, a similaridade entre ambas pode ser considerada baixa $\left(C_{m H}=0,473\right)$.

A área de monocultura apresentou maior riqueza de espécies (sistema lêntico: $37 \pm 1 \mathrm{IC}_{95 \%}$; sistema lótico: $42 \pm 1$ $\mathrm{IC}_{95 \%)}$ que a EEJ (sistema lêntico: $34 \pm 1 \mathrm{IC}_{95 \%}$; sistema lótico $\left.13 \pm 1 \mathrm{IC}_{95 \%}\right)$.

As variáveis físicas e químicas não apresentaram diferenças significativas entre as duas áreas estudadas $(t=1,16 ; \mathrm{p}>$ 0,05; $\mathrm{gl}=8$, para o sistema lótico e $\mathrm{t}=0,58 ; \mathrm{p}>0,05 ; \mathrm{gl}=4$, para o sistema lêntico).

\section{DISCUSSÃO}

Os impactos antrópicos, como a alteração de fluxo pelo represamento e a canalização, fragmentam e isolam os cursos d'água em relação a mata ciliar e as áreas de várzea, interrom- pendo o regime de perturbação natural e eliminando gradientes ambientais. Essas alterações diminuem a riqueza local porque homogeneizam os ambientes, diminuindo a disponibilidade de recursos e, criam ilhas de habitats que têm sua recolonização dependente da distância de outros habitats mais adequados (O'CONNOR 1991, ZWICK 1992, WARD 1998). Entretanto, a complexidade de ambientes pode tamponar o efeito da perturbação e os novos ambientes, criados pela ação de agentes perturbadores parecem ser favoráveis a muitas espécies de Odonata (SAMWAYS \& Steytler 1996, Stewart \& Samways 1998, Ferreira-Peruquetti \& de Marco Jr. 2002).

Neste trabalho, as variáveis físicas e químicas medidas nos corpos d'água parecem não ter sido suficientes para avaliar as diferenças observadas na comunidade de Odonata, sugerindo que as condições locais dos cursos d'água exercem grande influência na riqueza destes insetos (RichaRds et al. 1996) e que a estabilidade do córrego é mais importante na determinação da composição da comunidade que o uso do solo (DeATH \& WinTERBOURn 1994). Observações semelhantes foram feitas por LENAT \& CRAWFord (1994) em córregos dos EUA. Estes autores não encontraram diferenças entre parâmetros físicos e químicos em cursos d'água influenciados por diferentes usos do solo para explicar as diferenças encontradas por eles na estrutura das comunidades de macroinvertebrados. Para o Brasil, OмEто et al. (2000) não encontraram relação entre o uso do solo (áreas urbanizadas, pastagens e cana-de-açúcar) e a riqueza de macroinvertebrados, sugerindo que somente mudanças bruscas no uso do solo podem determinar as condições locais dos córregos. Apesar disso, vários estudos mostram que a intensidade do uso do solo pode alterar a estrutura do canal afetando a qualidade d'água em termos físicos e químicos, influenciando a distribuição da biota aquática (WARD \& STANFORD 1982, WeBsTer et al. 1983, Johnson et al.1997).

Apesar do histórico recente de regeneração, cerca de 40 anos (SANTos \& Pires 2000), a EEJ foi capaz de manter uma comunidade própria de Odonata e ser um reservatório potencial em espécies que podem migrar para colonizar ambientes adjacentes. Os ambientes protegidos da EEJ parecem favorecer espécies, talvez mais exigentes quanto ao habitat e que precisam, por exemplo, de ambientes com mata ciliar e corredeiras que, de outra forma, poderiam ser eliminadas regionalmente. Por outro lado, o isolamento das lagoas marginais, que foram formadas pelos meandros do rio Mogi-Guaçu, criaram ambientes com características bem diferentes dos amostrados fora da EEJ, possibilitando a formação de comunidades únicas na região.

No Brasil, a preferência das espécies de Odonata por habitat é pouco conhecida. Este trabalho amplia nosso conhecimento sobre a relação das espécies amostradas com o ambiente que exploram e, poderá servir de base para trabalhos futuros. Determinar as razões da preferência por habitat degradado por gêneros observados neste trabalho, como por exemplo Argia, Macrothemis e Oxyagrion são questões importantes, pois conhecer como esses insetos se reproduzem, utilizam o ambiente e respondem às atividades humanas é importante para sua utilização no monitoramento da qualidade ambiental dos sistemas aquáticos (Fore et al. 1996).

\section{AGRADECIMENTOS}

Agradecemos ao Programa BIOTA/FAPESP (processo $\mathrm{n}^{\circ}$ 99/11611-1) pelo auxílio financeiro, à Secretaria Estadual de Meio Ambiente do Estado de São Paulo pela permissão de 
coletas na EEJ, ao Frederico A. A. Lencioni pela identificação das espécies de Zygoptera e sugestões durante a condução do trabalho, à Profa. Dra. Janira M. Costa (Museu Nacional, UFRJ) pela confirmação e identificação de parte dos adultos de Anisoptera e ao Prof. Dr. Alcimar Carvalho (Museu Nacional,UFRJ) pela identificação das larvas; à Daniela Chaves Resende (UFV) pela identificação dos Erythrodiplax; à Dra. Adriana M. Z. C. Pires (UFSCar) pelo georeferenciamento dos pontos de coleta; ao Dr. Rui C. Peruquetti (UFSCar) pela edição do mapa, auxílio nas análises estatísticas e sugestões, à Renata Guereschi (UFSCar) por ter cedido a larva de Aeschnosoma.

\section{REFERÊNCIAS BIBLIOGRÁFICAS}

Allan, J.D. \& A.S. Flecker. 1993. Biodiversity conservation in running waters. Bioscience, Washington, D.C., 43 (1): 3243.

Allan, J.D. \& L.B. Johnson. 1997. Catchment-scale analysis of aquatic ecosystems. Freshwater Biology, London, 37:107111.

Carle, F.L. 1979. Environmental monitoring potential of the Odonata, with a list of rare and endangered Anisoptera of Virginia. United States. Odonatologica, Bilthoven, 8 (3): 319-323.

Cavalheiro, F.; M.V.R. Ballester; A.V. Krusche; S.A. Melo; J.L. Waechter; C.J. da Silva; M.C. D'Arienzo; M. Suzuki; R.L. Bozelli; T.P. Jesus \& J.E. Santos. 1990. Propostas preliminares referentes ao plano de zoneamento e manejo da Estação Ecológica de Jataí, Luiz Antônio, SP. Acta Limnologica Brasiliensia, Porto Alegre, 3: 951-968

Colwell, R. \& J. Coddington. 1994. Estimating terrestrial biodiversity through extrapolation. Philosophical Transactions of the Royal Society of London, Biology, London, 345: 101-118.

Colwell, R.K. 1997. EstimateS: Statistical estimation of species richness and shared species from samples. User's Guide and application published at: http://viceroy.eeb.uconn.edu/ estimates.

Corbet, P.S. 1980. A Biology of Odonata. Annual Review of Entomology, Palo Alto, 25: 189-217.

Death, R. \& M.J. Winterbourn. 1994. Environmental stability and community persistence: a multivariate perspective. Journal of North American Benthological Society, Lawrence, 13: 125-139.

Esteves, F.A. 1998. Fundamentos de Limnologia. Rio de Janeiro, Ed. Interciência, 602p.

Fore, L.S., J.R. Karr \& R.W. Wisseman. 1996. Assessing invertebrate responses to human activities: evaluating alternative approaches. Journal of North American Benthological Society, Lawrence, 15 (2): 212-231.

Ferreira-Peruquetti, P.S. \& P. De Marco Jr. 2002. Efeito da alteração ambiental sobre a comunidades de Odonata em riachos de Mata Atlântica de Minas Gerais, Brasil. Revista Brasileira de Zoologia, Curitiba, 19 (2): 317-327.

Gorham, C. \& D. Vodopich. 1992. Effects of acidic pH on predation rates and survivorship of damselfly nymphs. Hydrobiologia, Dordrecht, 242: 51-62.

GurTZ, E.G. \& J.B. WaLLACE 1984. Substrate-mediated response of stream invertebrates to disturbance. Ecology, Ithaca, 65: 1556-1569.
HeLtSHe, J. \& N. ForRester. 1983. Estimating species richness using the jackknife procedure. Biometrics, Arlington, 39: 1-11.

Johnson, L.B.; C. Richards; G. Host \& J.W. Arthur. 1997. Landscape influences on water chemistry in Midwest stream ecosystems. Freshwater Biology, London, 37: 193-208.

LENAT, D.R. \& J.K. CRAWFord. 1994. Effect of land use on water quality and aquatic biota of the three North Carolina Piedmont streams. Hydrobiologia, Dordrecht, 294: 185199.

Magurran, A.E. 1988. Ecological diversity and its measurement. London, Chapman and Hall, 179p.

Manly, B. 1991. Randomization and Monte Carlo methods in biology. London, Chapman and Hall, 281p.

- 1994. Multivariate statistical methods: a primer. London, Chapman and Hall, 215p.

O'ConNor, N.A. 1991. The effects of habitat complexity on the macroinvertebrates colonising wood substrates in a lowland stream. Oecologia, Berlin, 85: 505-512.

Ometo, J.P.H.B.; L.A. Martinelli; M.V. Ballester; AF. Gessner; A.V. Krusche; R.L. Victoria \& M. Williams. 2000. Effects of land use on water chemistry and macroinvertebrates in two streams of the Piracicaba river basin, south-east Brazil. Freshwater Biology, London, 44: 327-337.

Pires, A.M.Z.C.R.; J.E. Santos \& J.S.R. PIres. 2000. Caracterização ambiental e diagnóstico ambiental de uma unidade da paisagem. Estudo de caso: Estação Ecológica de Jataí e Estação Experimental de Luiz Antônio, p. 1-26, In: J.E. SANTos \& J.S.R. PIRES (Eds). Estudos Integrados em Ecossistemas. Estação Ecológica de Jataí. São Carlos, Rima, vol. 1, 346p.

RichaRds, C.; L.B. Johnson \& G.E. Host. 1996. Landscape-scale influences on stream habitats and biota. Canadian Journal of Aquatic Science, Ottawa, 53: 295-311.

Samways, M.J. \& N.S. Steytler. 1996. Dragonfly (Odonata) distribution patterns in urban and forest landscapes, and recommendations for riparian management. Biological Conservation, London, 78: 279-288.

Santos, J.E. \& J.S.R. Pires. 2000. Estudos Integrados em Ecossistemas. Estação Ecológica de Jataí. São Carlos, Rima, vol. 1, 346p.

São Paulo. 1985. Áreas naturais do Estado de São Paulo. São Paulo, Conselho Estadual do Meio Ambiente. 168p.

. 1997. Cerrado: Bases para conservação e uso sustentável das áreas de cerrado do Estado de São Paulo. Série Probio/São Paulo. São Paulo, Secretaria de Estado do Meio Ambiente, 113p.

SCHміDт, E. 1985. Habitat inventarization, characterization and bioindication by a Representative Spectrum of Odonata Species (RSO). Odonatologica, Bilthoven, 14 (2): 127-133.

Setzer, J. 1966. Atlas climatológico do estado de São Paulo. Comissão Interestadual da Bacia do Paraná-Paraguai. São Paulo, CESP, 40p.

Sponseller, R.A.; E.F. Benfield \& H.M. VAllet. 2001. Relationships between land use, spatial scale and stream macroinvertebrate communities. Freshwater Biology, London, 46: 1409-1424.

Stewart, D.A.B. \& M.J. SAmways. 1998. Conserving dragonfly (Odonata) assemblages relative to river dynamics in an african savanna game reserve. Conservation Biology, Seattle, 12 (3): 683-692.

WARD, J.V. 1998. Riverine landscapes: biodiversity patterns, 
disturbance regimes, and aquatic conservation. Biological Conservation, London, 83(3): 269-278.

Ward, J.V. \& J.A. STANFord. 1982. Thermal responses in the evolutionary ecology of aquatic insects. Annual Review of Entomology, Palo Alto, 27: 97-117.

Webster, J.R.; M.E. Gurtz; J.J. Hains; J.L. Meyer; W.T. Swank; J.B. Waide \& J.B. Wallace. 1983. Stability of stream ecosystems, p. 355-394. In: J.B. Barnes \& G.W. Minshall (Eds). Stream Ecology. New York, Plenum Press, 412p.

Wolda, H. 1983. Diversity, diversity indices and tropical cockroaches. Oecologia, Berlin, 58: 290-298.

Zwick, P. 1992. Stream habitat fragmentation - a threat to biodiversity. Biodiversity Conservation, Dordrecht, 1: 8097.

Recebido em 05.XII.2002; aceito em 07.V.2003.

Revista Brasileira de Zoologia 20 (2): 219-224, junho 2003 